\title{
Surveillance for Lyme Disease in Canada, 2009-2012
}

\author{
Jules Koffi ${ }^{\star 1}$, Robbin Lindsay ${ }^{3}$ and Nicholas Ogden ${ }^{2}$ \\ ${ }^{1}$ Centre for Food-Borne, Environmental and Zoonotic Infectious Diseases, Public Health Agency of Canada, Saint-Hyacinthe, QC, \\ Canada; ${ }^{2}$ National Microbiology Laboratory, Public Health Agency of Canada, Saint-Hyacinthe, QC, Canada; ${ }^{3}$ National Microbiology \\ Laboratory, Public Health Agency of Canada, Winnipeg, MB, Canada
}

\section{Objective}

To summarize the first 4 years (2009-2012) of national surveillance for LD in Canada and to conduct a preliminary comparison of presenting clinical manifestations in Canada and the United-States.

\section{Introduction}

LD, caused by Borrelia burgdorferi in North America is transmitted to humans from wild animal reservoir hosts by Ixodes spp. ticks ${ }^{1}$ in their woodland habitats ${ }^{2}$. LD risk in Canada occurs where tick vectors are established in southern British Columbia (I. pacificus) and in southern parts of central and eastern Canada where I. scapularis is spreading from the United States (US) ${ }^{3}$. LD became nationally notifiable in Canada in 2009 and demographic data on human cases is submitted by provinces to Canadian Notifiable Disease Surveillance System of the Public Health Agency of Canada (PHAC). A Lyme Disease Enhanced Surveillance system was initiated by PHAC in 2010 to obtain more detailed data on LD cases. These surveillance systems aim to identify changing trends in LD incidence, the population at risk and the types of clinical disease in Canada.

Surveillance data for 2009-2012 are analyzed to describe the early patterns of LD emergence in Canada. Patterns of LD cases (age, season of acquisition and presenting manifestations) were compared against those reported in the US.

\section{Methods}

The numbers and incidence of reported cases by province, month, year and sex were calculated. Logistic regression was used to examine trends over time. Acquisition locations were mapped and presenting clinical manifestations reported for jurisdictions where data was available. Variations by province, year, age and sex as well as presenting clinical symptoms were explored by logistic regression. An initial comparative analysis was made of presenting symptoms in Canada and the US.

\section{Results}

The numbers of reported cases rose significantly from 144 in 2009 to 338 in 2012 (coefficient $=0.34$, standard error $=0.07, \mathrm{P}$ $<0.05$ ), mostly due to an increased incidence of infections acquired in Canada. Most cases occurred in locations where vector tick populations were known to be present. Incidence was highest in adults aged 55 to 74 years and in children aged 5 to 14 years. Most cases (95\%) were acquired from April to November. Of cases acquired in endemic areas, $39.7 \%$ had manifestations of early LD, while $60.3 \%$ had manifestations of disseminated LD. The proportion of cases acquired in endemic areas presenting with early LD was lower than that reported in the US.

\section{Conclusions}

LD incidence is increasing in Canada. Most cases are acquired where vector tick populations are spreading and this varies geographically within and among provinces. The lower proportion of cases presenting with early LD in Canada compared with the US suggests lower awareness of early LD in Canada, but this requires further study.

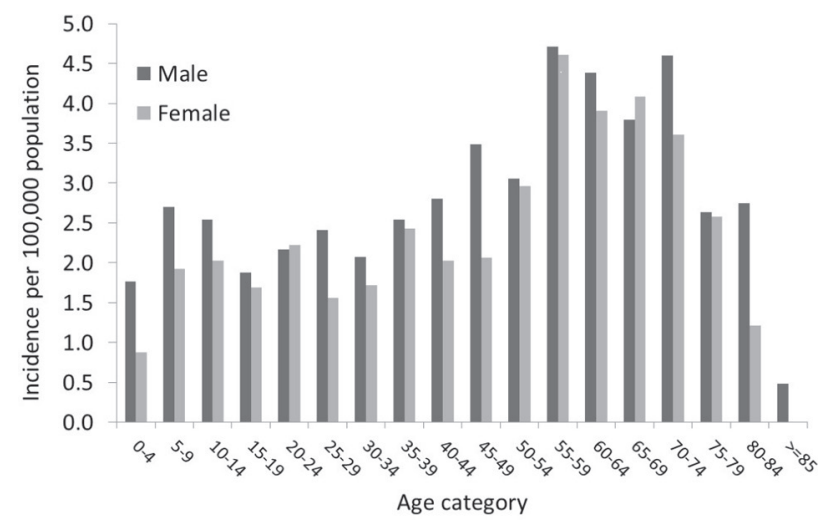

Figure: The incidence of reported LD cases per 100,000 population during 2009-2012 by age and sex.

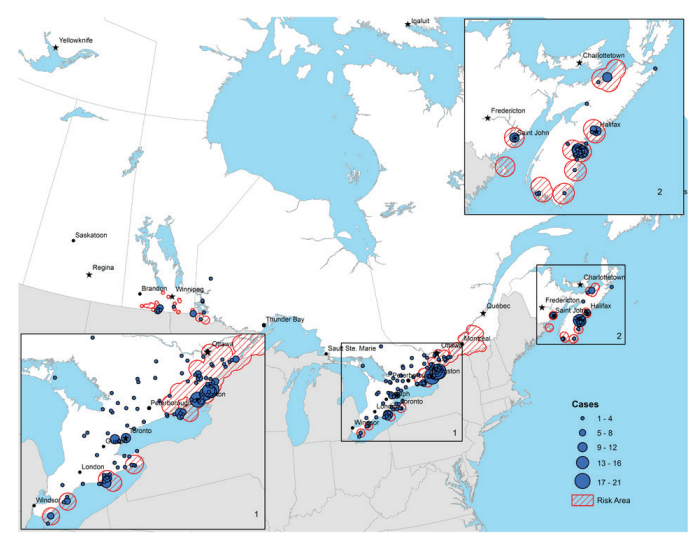

Figure: The reported location of acquisition of LD cases acquired in Canada from 2009-2012

\section{Keywords}

Lyme disease; Surveillance; tick-borne disease

\section{References}

1. Ogden NH, Lindsay LR, Morshed M, Sockett PN, Artsob H. The emergence of Lyme disease in Canada. Can.Med.Assoc.J. 2009 Jun 9;180(12):1221-4.

2. Kurtenbach K, Hanincova K, Tsao JI, Margos G, Fish D, Ogden $\mathrm{NH}$. Fundamental processes in the evolutionary ecology of Lyme borreliosis. Nat.Rev.Microbiol 2006 Sep;4(9):660-9.

3. Ogden NH, Koffi JK, Pelcat Y, Lindsay LR. Environmental risk from Lyme disease in central and eastern Canada: a summary of recent surveillance information. Can.Commun.Dis.Rep. 2014 Jun $3 ; 40(5): 74-82$.

\section{*Jules Koffi}

E-mail: jules.konan.koffi@phac-aspc.gc.ca

ISDS Annual Conference Proceedings 2015. This is an Open Access article distributed under the terms of the Creative Commons AttributionNoncommercial 3.0 Unported License (http://creativecommons.org/licenses/by-nc/3.0/), permitting all non-commercial use, distribution, and reproduction in any medium, provided the original work is properly cited. 